\title{
Consumo de Brugmansia suaveolens (Trombeta de Anjo) e perturbação psíquica
}

\author{
Consumption of Brugmansia suaveolens (Angel's Trumpet) and psychic perturbation \\ Consumo de Brugmansia suaveolens (Trompeta de Ángel) y perturbación psíquica
}

Recebido: 19/12/2020 | Revisado: 27/12/2020 | Aceito: 01/01/2021 | Publicado: 04/01/2021

Eduardo de Sousa Martins e Silva ORCID: https://orcid.org/0000-0001-6257-4681 Universidade Federal do Triângulo Mineiro, Brasil E-mail: eduardosousa25@gmail.com

Ben Hur Vitor Silva Ono

ORCID: https://orcid.org/0000-0002-3911-5361 Universidade para o Desenvolvimento do Estado e da Região do Pantanal, Brasil E-mail: benhur_ono@hotmail.com

Bruno Massayuki Makimoto Monteiro

ORCID: https://orcid.org/0000-0003-1258-4878 Universidade Estadual de Mato Grosso do Sul, Brasil E-mail: brunoftmakimoto@hotmail.com

Joaquim Borges de Menezes Neto ORCID: https://orcid.org/0000-0001-6603-9133 Universidade Federal de Mato Grosso do Sul, Brasil E-mail: joaquim.paranhos4@gmail.com José Carlos Souza

ORCID: https://orcid.org/0000-0003-4460-3770 Universidade Estadual de Mato Grosso do Sul, Brasil E-mail: josecarlossouza@uol.com.br

\begin{abstract}
Resumo
A planta Brugmansia suaveolens (Humb. \& Bonpl. ex Willd.) Sweet, conhecida popularmente como "Trombeta de Anjo", é típica de regiões tropicais e sul americanas, incluindo o Brasil, e é famosa por suas propriedades alucinógenas, perturbadoras, sedativas, entre outras. Neste contexto, é utilizada em práticas ritualísticas, curativas e recreacionais. Dessa maneira, o presente estudo tem como objetivo analisar e discutir o consumo de B. suaveolens e o seu potencial de perturbação psíquica. Fez-se uma revisão bibliográfica integrativa, utilizando-se de artigos dos últimos 10 anos (2010-2020), com os descritores "Brugmansia suaveolens", "Toxinas", "Alucinações", "Delirium", "Síndrome Anticolinérgica Aguda", "Escopolamina", "Atropina", "Chá de Trombeta", "Trombeta de Anjo", "Mududu". A Brugmansia suaveolens apresenta propriedades antinociceptiva, nematicida e relaxante muscular e a toxicidade do seu consumo é dose dependente. Neste sentido, concentrações maiores de flores na infusão de água refletem em maiores concentrações de atropina e escopolamina, os quais inibem os receptores muscarínicos. Esta inibição é responsável pela toxicidade anticolinérgica, causando febre, alucinações, confusão, delírio, dentre outros sintomas. Conclui-se que a utilização de $B$. suaveolens apresenta riscos, devido ao seu alto potencial alucinógeno, e deve ser visto, portanto, com importância clínica.
\end{abstract}

Palavras-chave: Toxinas; Alucinações; Delirium; Síndrome anticolinérgica aguda; Escopolamina; Atropina.

\begin{abstract}
The Brugmansia suaveolens (Humb. \& Bonpl. ex Willd.) Sweet plant, popularly known as "Angel's Trumpet ", is typical of tropical and South American regions, including Brazil, and is famous for its hallucinogenic, disturbing, sedative, among others. In this context, it is used in ritualistic, curative and recreational practices. Thus, the present study aims to analyze and discuss the consumption of B. suaveolens and its potential for psychic disturbance. An integrative bibliographic review was made, using articles from the last 10 years (2010-2020), with the descriptors "Brugmansia suaveolens", "Toxins", "Hallucinations", "Delirium", "Acute Anticholinergic Syndrome", "Scopolamine", "Atropine", "Trumpet Tea", "Angel’s Trumpet", "Mududu". B. suaveolens has antinociceptive, nematicide and muscle relaxant properties and the toxicity of its consumption is dose dependent. In this sense, higher concentrations of flowers in water infusion reflect higher concentrations of atropine and scopolamine, which instill muscarinic receptors. This inhibition is responsible for anticholinergic toxicity, causing fever, hallucinations, confusion, delirium, among other symptoms. It is concluded that the use of B. suaveolens presents risks, due to its high hallucinogenic potential, and should be seen, therefore, with clinical importance.
\end{abstract}

Keywords: Toxins; Hallucinations; Delirium; Anticholinergic syndrome; Scopolamine; Atropine. 


\section{Resumen}

La planta Brugmansia suaveolens (Humb. \& Bonpl. ex Willd.) Sweet, conocida popularmente como "Trombeta de Anjo", es típica de las regiones tropicales y sudamericanas, incluyendo Brasil, y es famosa por su alucinógeno, perturbador, sedante, entre otros. En este contexto, se utiliza en prácticas ritualistas, curativas y recreativas. Por lo tanto, el presente estudio tiene como objetivo analizar y discutir el consumo de B. suaveolens y su potencial de perturbación psíquica. Se hizo una revisión bibliográfica integradora, utilizando artículos de los últimos 10 años (2010-2020), con los descriptores "Brugmansia suaveolens", "Toxinas", "Alucinaciones", "Delirium", "Síndrome Anticolinérgico Agudo", "Escopolamina", "Atropina", "Trompeta de Ángel", "Muduu". B. suaveolens tiene propiedades antinociceptivas, nematicidas y relajantes musculares y la toxicidad de su consumo depende de la dosis. En este sentido, mayores concentraciones de flores en la infusión de agua reflejan mayores concentraciones de atropina y escopolamina, que inculcar receptores muscarínicos. Esta inhibición es responsable de la toxicidad anticolinérgica, causando fiebre, alucinaciones, confusión, delirio, entre otros síntomas. Se concluye que el uso de $B$. suaveolens presenta riesgos, debido a su alto potencial alucinógeno, y debe verse, por lo tanto, con importancia clínica.

Palabras clave: Toxinas; Alucinaciones; Delirium; Síndrome anticolinérgico agudo; Escopolamina; Atropina.

\section{Introdução}

O dilema do uso, abuso, dependência, entre outros conceitos relacionados às drogas psicoativas lícitas e ilícitas, por vezes, impede que políticas públicas sociais e de saúde possam ser eficientes o suficiente para diminuírem os índices alarmantes de pessoas com idade cada vez mais tenra envolvidas com álcool e outras drogas. Isto porque muitas classificações diagnósticas de transtornos mentais são contraditórias ao seguirem um conceito mais categorial e outras mais dimensional. Este último com o propósito de envolver várias nosologias e conceitos em um espectro; ambas as Escolas Psicopatológicas Categorial e Dimensional são sujeitas a críticas e sugestões de aprimoramento. Porém, o atraso de um diagnóstico, ou mesmo de um tratamento, que, naturalmente começam com a discussão aberta dos efeitos do consumo dessas substâncias, faz com que novos indivíduos sejam afetados por transtornos mentais cada vez mais grave (Gaebel, Stricker \& Kerst, 2020).

Quando se tratam de drogas derivadas de plantas em geral, a situação se agrava, pois se esbarram em conceitos e princípios religiosos e culturais, que na maioria das vezes acobertam já um quadro clínico mais sério ocasionado pela farmacologia das substâncias encontradas. Por conta disto, há de se ter uma visão holística do ser humano, levando-se em conta os seus aspectos fisiológicos, emocionais, sociais, ambientais, históricos, culturais e espirituais no sentido de crenças. Nenhum destes é mais importante do que o outro, pois são diferentes, entretanto complementares na vida humana. As drogas psicoativas interferem em todos estes tópicos; quando se tem à frente um elemento da flora, componente de uma planta como a Brugmansia suaveolens (Solanaceae), é essencial uma abordagem clara e integral do usuário. Neste contexto, o objetivo deste estudo é discutir os aspectos relacionados à farmacologia, padrões de consumo e perturbações psíquicas relacionadas a esta planta, popularmente conhecida como "Trombeta de Anjo", "Zabumba", "Saia-branca", entre outros (Souza, Portela \& Marques, 2019). Sua utilização, principalmente por meio do chá de suas flores e de suas folhas, tem alto potencial de causar delírios e alterações importantes nos níveis de consciência (Jayawickreme, Janaka \& Subasinghe, 2019). Dessa forma, sendo uma planta componente da flora brasileira e de outras regiões sul-americanas, a discussão de efeitos tóxicos relacionados ao seu consumo é de grande relevância (Mesquita, Pinto \& Moreira, 2017).

\section{Método}

Foi feito um estudo qualitativo, com uma revisão bibliográfica descritiva e exploratória, do tipo integrativa. Cabe ressaltar que a revisão bibliográfica do tipo integrativa é um método que reúne, avalia e sintetiza os resultados de levantamentos e pesquisas sobre uma temática específica. Nesta categoria de revisão, seguem-se certas etapas; as aqui percorridas foram a elaboração da pergunta de pesquisa, amostragem ou busca na literatura dos estudos primários, extração de dados, avaliação dos estudos primários incluídos, interpretação dos resultados, e apresentação da revisão (Mendes, Silveira \& 
Galvão, 2008). Para a estruturação da pergunta de pesquisa desta revisão integrativa, utilizou-se a estratégia PICO (acrônimo para patient, intervention, comparison, outcomes). Esta possibilita a identificação de palavras-chave, as quais auxiliam na localização de estudos primários relevantes nas bases de dados (Stillwell et al., 2010).

Sendo assim, a questão de pesquisa foi: "Qual é a relação entre o consumo de B. suaveolens e perturbação psíquica?" Nela, adaptando-se à estratégia PICO, o primeiro elemento foi a planta B. suaveolens (conhecida popularmente como "Trombeta de Anjo"); o segundo perturbação psíquica; o terceiro elemento se a referida planta causa confusão psíquica e o quarto se esta perturbação é temporária ou definitiva. Ressalta-se que, dependendo do método de revisão, não se emprega todos os elementos da estratégia PICO.

A busca dos estudos primários ocorreu dos artigos publicados nos últimos 10 anos (2010-2020), em português, em inglês e em espanhol. Foram utilizados os bancos de dados bibliográficos National Library of Medicine (PubMed), Scientific Electronic Library Online (SciELO), Latin American and Caribbean Health Sciences Literature (Lilacs), entre os descritores usaram-se: "Brugmansia suaveolens", "Toxinas", "Alucinações", "Delirium", "Síndrome Anticolinérgica Aguda", "Escopolamina", "Atropina", "Chá de Trombeta", "Trombeta de Anjo", "Mududu” e o booleano "AND” ou "E”. Foram adotados os seguintes critérios de inclusão: a) artigos originais (estudos experimentais, ensaios clínicos randomizados, estudos caso-controle); b) artigos publicados na língua inglesa, na língua portuguesa e na língua espanhola; c) artigos publicados no ano de 2010 até 2020; d) artigos que possuíam no título e/ou resumo os descritores utilizados na busca.

A pesquisa foi concluída em Setembro de 2020 e, no total, foram obtidos 21 artigos científicos. Foi realizada uma leitura dinâmica dos resumos de cada artigo que se enquadrasse nos critérios de inclusão e, em seguida, foram selecionados apenas aqueles que poderiam contribuir com o objetivo do estudo. Por fim, 12 publicações prosseguiram para uma leitura minuciosa, com a finalidade de extrair os dados de cada pesquisa e, posteriormente, estas foram categorizadas de acordo com os tópicos de interesse para a presente revisão. Em seguida, procedeu-se a discussão dos resultados.

\section{Resultados e Discussão}

\subsection{Classificação, conceitos e histórico da Brugmansia suaveolens}

\subsubsection{Descrição}

A Brugmansia suaveolens (Humb. \& Bonpl. ex Willd.) Sweet, é uma planta da família Solanaceae, e tem sua origem em regiões tropicais da América do Sul, inclusive no Brasil, em florestas de planície, margens de rios e zonas urbanas. É uma árvore de folhas ovais com 1,8 a 4,6 metros de altura, até $25 \mathrm{~cm}$ de comprimento e 15,2 cm de largura, atingindo um tamanho maior quando cresce na sombra. Suas flores são bonitas, com cheiro adocicado, de aproximadamente $30 \mathrm{~cm}$ de comprimento e em formato de trompete; a sua cor pode ser amarela, laranja, creme ou rosa, mas usualmente são brancas (Lusweti et al., 2011).

\subsubsection{História e utilização}

A B. suaveolens já foi utilizada na sedação das vítimas de sacrifícios em certas cerimônias espirituais, além de ser um agente anestésico para uso tópico (como abordado no item 3.3). Na atualidade é cultivada nos jardins, como também pode ser inalada, ingerida como comida ou na forma de chá, ou administrada via enema. Em alguns lugares, como na Tanzânia, esta planta é adicionada na cerveja; em outros, certos curandeiros tratam algumas doenças após desidratarem as suas folhas e adicionarem ao tabaco (Lusweti et al., 2011). Além disso, devido aos seus efeitos alucinógenos, seu baixo custo e fácil demanda, ela também tem sido muito usada para fins recreacionais (Jayawickreme, Janaka \& Subasinghe, 2019). 


\subsection{Farmacologia}

São identificados na composição da B. suaveolens tanto aminas, carboidratos, alcalóides, compostos fenólicos, esteróides, terpenóides, taninos, glicosídeos, como também antraquinônicos, saponinas e triterpenos. É variável a concentração de cada um destes componentes, levando-se em consideração as suas flores vivas; entretanto, há constantes mudanças no perfil dos seus componentes voláteis, os quais vão depender tanto dos fatores intrínsecos (genéticos), como extrínsecos (luz, temperatura e estresse hídrico). Quando as suas flores são cortadas, elas sofrem uma deterioração rápida e perdem os seus compostos voláteis; além desses, outros fatores também afetam, como é o caso dos ataques de patógenos como vírus, bactérias, fungos e nematóides, além de seres herbívoros (Petricevich et al., 2020).

$\mathrm{Na}$ revisão realizada por Petricevich et al., constataram-se diversos compostos com atividade farmacológica e toxicológica, distribuídas por toda a estrutura da B. suaveolens, sendo elas: nas flores maduras e imaturas, nas frutas imaturas, na corola, nas raízes e no néctar da Trombeta de anjo já foram identificados 59 alcalóides; já nas suas folhas e flores encontraram-se 50 compostos voláteis, mais presentes nas flores. Também suas flores e folhas são compostas por agentes fenólicos glicosilados, cumarina, 7 flavonóides e 3 esteroides; enquanto quatro hidrocarbonetos foram encontrados apenas nas flores desta planta (Petricevich et al., 2020). Todos esses compostos encontrados na B. suaveolens têm vários efeitos cientificamente comprovados, que a seguir são descritos:

\subsection{Atividade antinociceptiva}

A atividade antinociceptiva consiste na anulação ou redução da percepção e transmissão de estímulos dolorosos. Existem os chamados nociceptores, que são terminações nervosas livres as quais respondem a estímulos nociceptivos; assim como, estes detectam lesão nos tecidos; eles podem ser mecânicos, térmicos e químicos. O potencial antinociceptivo de uma planta pode ser medido pelo seu poder de aumentar o limiar de excitação destes nociceptores à dor, ou fazer com que estes não percebam ou não respondam ao estímulo doloroso promovido (Silva et al., 2013). Foi observada atividade antinociceptiva nas doses de 100 e $300 \mathrm{mg} / \mathrm{Kg}$, a partir da extração aquosa das flores da B. suaveolens (Petricevich et al., 2020). Para esse estudo foram utilizados ratos, sobre os quais foram aplicados estímulos térmicos e químicos de nocicepção. Acredita-se que o efeito nociceptivo dessa planta seja devido à ação em receptores benzodiazepínicos (Muccillo-Baisch et al., 2010).

\subsection{Atividade nematicida}

A atividade nematicida refere-se à capacidade da planta ou produto químico de eliminar nematoides parasitas (Mesquita, Pinto \& Moreira, 2017; Moreira, 2013). Foi demonstrado que a extração etanólica das flores da B. suaveolens, na concentração de $1000 \mathrm{mg} / \mathrm{L}$, apresenta 64\% de atividade nematicida contra Meloidogyne incognita. Outro estudo, com a extração etanólica de flores e caules, numa diluição de $12,5 \mathrm{mg} / \mathrm{ml}$, demonstrou $100 \%$ de atividade larvicida contra Ancylostoma spp (Petricevich et al., 2020). As substâncias responsáveis por esse efeito foram alcaloides tropanos, dentre eles hiosciamina, atropina e escopolamina, sendo essa última a principal responsável pela atividade nematicida, como foi demonstrado por um terceiro estudo, que analisava os efeitos da B. suaveolens, a partir de seu extrato metanólico, contra Meloidogyne sp. A letalidade, foi constatado, ainda, atingiu níveis entre 90 e 100\% contra espécies como Hoplolaimus indicus, Helicotylenchus multicinctus e Meloidogyne incógnita (Salazar-Anton \& Guzman-Hernandez, 2014).

\subsection{Citotoxicidade}

Chama-se de citotoxidade a propriedade nociva de uma substância, como por exemplo uma planta, em relação às células (Marinho et al., 2017). Estudos com o extrato aquoso da B. suaveolens obtiveram um LD50 (dose letal mediana) na concentração de $106 \mu \mathrm{g} / \mathrm{mL}$ (Petricevich et al., 2020). Um ensaio de letalidade com Artemia sp., em inglês conhecido como 
"brine shrimp lethality bioassay" foi utilizado a fim de serem alcançados os resultados obtidos. Todos os ensaios foram refeitos três vezes (Bussmann et al., 2011).

\subsection{Relaxante muscular}

Um relaxante muscular é uma planta ou fármaco que afeta o músculo esquelético, diminuindo o seu tônus. A extração etanólica da planta B. suaveolens possui atividade de relaxamento muscular, em uma concentração de $75.5 \mathrm{~g} / \mathrm{mL}$ (Petricevich et al., 2020). Para a realização desse estudo foram utilizados coelhos brancos Nova Zelância machos. Acredita-se que a escopolamina esteja por trás do efeito de relaxante muscular, devido à sua ação anticolinérgica (Encarnación-Dimayuga, Altamirano, \& Maki, 1998).

\subsection{Informações adicionais}

Ainda sobre a toxicidade da B. suaveolens, foi demonstrado que diversos fatores (climáticos, sazonais, entre outros) influenciam na concentração de alcalóides, que está diretamente relacionado aos seus efeitos tóxicos. Entre os compostos, os com o maior potencial de toxicidade são a atropina e a escopolamina. Ressalta-se, no entanto, que estas substâncias, em dadas doses, também têm utilização terapêutica. Além disso, as partes da planta mais relacionadas ao intoxicamento são as flores (77.5\%), folhas (13.4\%), frutas (4.5\%) e raiz (2.3\%) (Barreto, 2016).

\subsection{Alterações psíquicas da Brugmansia suaveolens}

A ingestão de B. suaveolens traz repercussões em diversos sistemas do organismo humano, tornando importante o conhecimento dos mecanismos e suas consequências de maneira detalhada, haja vista que é uma planta consumida para fins anestésicos, eufóricos e alucinógenos. Desta maneira, o início dos sintomas, como relatado em outros estudos é dose dependente e se dá entre 15 minutos ( 1 copo de infusão de 4 flores/ 1 Litro de água) e 30 minutos ( $350 \mathrm{~mL}$ ingeridos de infusão de água com número de flores desconhecidos), podendo ser alucinações, euforia, confusão, taquicardia, hipertemia, retenção urinária, febre, hipertensão arterial, arritmia, midríase, xerostomia, tontura e delírio (Jayawickreme, Janaka \& Subasinghe, 2019; Barreto, 2016).

A psicopatologia da ingestão de $B$. suaveolens é centrada em alcalóides tropânicos, principalmente a hioscina, a escopolamina e a atropina (Barreto, 2016; Doan et al., 2019). Tais alcalóides induzem inibição da atividade de receptores muscarínicos no sistema nervoso central e periférico, resultando em toxicidade anticolinérgica e como consequência sintomas, tais quais a confusão, delírio, alucinações, visão embaçada, midríase, retenção urinária, febre, colapso cardiorrespiratório, coma e morte (Soulaidopoulos et al., 2017). Ressalta-se, com base nisto, que os efeitos psicopatológicos são temporários e dependentes do efeito das toxinas no organismo. Não obstante, conforme reportado por Lakstygal et al. (2019), a utilização de plantas ricas em alcaloides, como escapolamina e atropina, podem, também, induzir estados de psicose aguda, necessitando de assistência médica imediata.

\subsection{Conduta médica no manejo do indivíduo intoxicado pela Brugmansia suaveolens}

De acordo com a Organização Mundial de Saúde (OMS), define-se intoxicação aguda como um quadro que se estabelece após a utilização/administração de uma substância psicoativa, ocasionando perturbações nos níveis de julgamento, cognição, consciência, comportamento e nas funções fisiológicas do usuário. Dentro deste escopo, a intoxicação pela planta B. suaveolens gera a Síndrome Anticolinérgica Aguda, devido ao efeito de toxinas, como a escopolamina e a atropina (Lakstygal et al., 2018). Em vista disso, a conduta médica no tratamento do indivíduo em intoxicação aguda pela planta deve ser pautado 
na estabilização de seus sinais vitais (cuidados de suporte), no uso de carvão ativado e, caso necessário, na utilização de antídoto para Síndrome Anticolinérgica (Dawson \& Buckley, 2016).

Nos pacientes com ingestão inferior a uma hora ( $<60$ mins), pode-se administrar carvão ativado, a fim de minimizar a absorção das toxinas (G., O’Malley \& R., O’Malley, 2020). O antídoto para a Síndrome Anticolinérgica Aguda é a fisostigmina. Ela é um inibidor de acetilcolinesterase, com atividade em todo o Sistema Nervoso (G., O’Malley \& R., O’Malley, 2020). Ressalta-se que a maioria dos pacientes podem ser tratados sem a necessidade do seu uso. No entanto, em casos de taquiarritmia, com comprometimento hemodinâmico, agitação severa ou psicose, a utilização da fisostigmina é aconselhada (The Royal Children's Hospital Melbourne, 2017). Devido ao potencial que a planta B. suaveolens tem de causar agitação e perturbação psíquica, o uso de benzodiazepínicos é de relevante aplicação no controle destes sintomas (Ramnarine $\&$ Ahmad, 2020).

\section{Considerações Finais}

Mesmo com toda a evolução tecnológica da farmacologia e farmacogenética, a medicina ainda se ocupa de muitas plantas e outros produtos da flora mundial. Estas são oriundas dos mais diversos tipos de biomas e territórios. Entretanto, seus efeitos farmacodinâmicos e farmacocinéticos podem ser utilizados, também, para fins não medicinais e não terapêuticos. A $B$. suaveolens, como exemplo, pode ter repercussões físicas, psíquicas e sociais diversas. Todavia, ainda não há uma regulamentação do seu plantio e da sua manipulação. Os seus efeitos, mesmo com promissor potencial, em mãos que não têm um conhecimento adequado de seu manejo, podem se tornar uma droga com grande potencial de intoxicação. Entre as complicações psiquiátricas, há a possibilidade de uma diagnóstico diferencial, com estados confusionais e psicóticos, havendo a necessidade de uma formação clínica adequada dos profissionais de saúde, a fim de um diagnóstico adequado, visando a melhor terapêutica possível e um prognóstico adequado.

\section{Referências}

Barreto, S. A. (2016). Intoxicación por uso recreativo de 'floripondio', reporte de caso. Revista Médica de Risaralda, 22(1), 71-72.

Bussmann, R. W., Malca G., Glenn, A., Sharon, D., Nilsen, B., Parris, B., \& Townesmith, A. (2011). Toxicity of medicinal plants used in traditional medicine in Northern Peru. Journal of Ethnopharmacology, 137(1), 121-140.

Dawson, A. H., \& Buckley, N. A. (2016). Pharmacological management of anticholinergic delirium - theory, evidence and practice. British journal of clinical pharmacology, 81(3), 516-524. https://doi.org/10.1111/bcp.12839.

de Souza, G. L. S., Portela, C. R., \& Marques, D. D. (2019). O uso da espécie Brugmansia suaveolens (Solanaceae) como ornamental e na medicina popular. Scientia Naturalis, 1(1).

Doan, U. V., Wu, M. L., Phua, D. H., Mendez Rojas, B., \& Yang, C. C. (2019). Datura and Brugmansia plants related antimuscarinic toxicity: an analysis of poisoning cases reported to the Taiwan poison control center. Clinical toxicology (Philadelphia, Pa.), 57(4), 246-253. https://doi.org/10.1080/15563650.2018.1513527.

Encarnación-Dimayuaga, R., Altamirano, L., \& Maki, K. A. (1998). Screening of Medical Plants from Baja California Sur (Mexico) by Their Effects on Smooth Muscle Contractility. Pharmaceutical Biology, 36(2), 124-130.

Gaebel, W., Stricker, J., \& Kerst, A. (2020). Changes from ICD-10 to ICD-11 and future directions in psychiatric classification neuroscience, 22(1), 7-15. https://doi.org/10.31887/DCNS.2020.22.1/wgaebel.

Jayawickreme, K. P., Janaka, K., \& Subasinghe, S. (2019). Unknowing ingestion of Brugmansia suaveolens leaves presenting with signs of anticholinergic toxicity: a case report. Journal of medical case reports, 13(1), 322. https://doi.org/10.1186/s13256-019-2250-1.

Lusweti, A., Wabuyele, E., Ssegawa, P., \& Mauremootoo, J. (2011). Brugmansia suaveolens (Angel's trumpet). Brugmansia suaveolens (Angel's trumpet). <https://keys.lucidcentral.org/keys/v3/eafrinet/weeds/key/weeds/Media/Html/Brugmansia_suaveolens_(Angels_Trumpet).htm>.

Lakstygal, A. M., Kolesnikova, T. O., Khatsko, S. L., Zabegalov, K. N., Volgin, A. D., Demin, K. A., Shevyrin,V.A., Wappler-Guzzetta, E.A., \& Kalueff, A. V. (2018). DARK Classics in Chemical Neuroscience: Atropine, Scopolamine, and Other Anticholinergic Deliriant Hallucinogens. ACS chemical neuroscience, 10(5), 2144-2159. 
Research, Society and Development, v. 10, n. 1, e11610111366, 2021

(CC BY 4.0) | ISSN 2525-3409 | DOI: http://dx.doi.org/10.33448/rsd-v10i1.11366

Marinho, J. C. S., de Almeida Cunha, L., Lacerda, M. F. L. S., Lacerda, G. P., Coelho, R. G., \& Girelli, C. F. M. (2017). Avaliação comparativa da citotoxicidade dos cimentos MTA Fillapex e AH Plus: revisão integrativa. Arquivos em Odontologia, 53.

Mesquita, M. O. M. D., Pinto, T. M. F., \& Moreira, R. F. (2017). Potencial antimicrobiano de extratos e moléculas isolados de plantas da Caatinga: uma revisão. Revista Fitos, Vol. 11(2), 119-249. https://doi.org/10.5935/2446-4775.20170028.

Moreira, L. C. B. (2013). Ação nematicida do eugenol em tomateiro. Pesqui. Agropecu. Trop, 286-291. https://doi.org/10.1590/S1983-40632013000300011.

Mendes, K. D. S., Silveira, R. C. D. C. P., \& Galvão, C. M. (2008). Revisão integrativa: método de pesquisa para a incorporação de evidências na saúde e na enfermagem. Texto \& contexto enfermagem, 17(4), 758-764.

Muccillo-Baisch, A. L., Parker, A. G., Cardoso, G. P., Cezar-Vaz, M. R., \& Flores Soares, M. C. (2010). Evaluation of the Analgesic Effect of Aqueous Extract of Brugmansia suaveolens Flower in Mice: Possible Mechanism Involved. Biological Research For Nursing, 11(4), 345-350.

O’Malley, G.F., \& O’Malley, R. (2020). General Principles of Poisoning. Kenilworth: MSD Manuals. <https://www.msdmanuals.com/enpt/professional/injuries-poisoning/poisoning/general-principles-of-poisoning>.

Petricevich, V. L., Salinas-Sánchez, D. O., Avilés-Montes, D., Sotelo-Leyva, C., \& Abarca-Vargas, R. (2020). Chemical Compounds, Pharmacological and Toxicological Activity of Brugmansia suaveolens: A Review. Plants, 9(9), 1161.

Ramnarine, M., \&Ahmad, D. A. (2020). Anticholinergic Toxicity Treatment \& Management. New York: Vearrier D. $<$ https://emedicine.medscape.com/article/812644-treatment\#d10>.

Salazar-Anton, W., \& Guzman-Hernandez, T., J. (2014). Efecto nematicida de extractos de Quassia amara y Brugmansia suaveolens sobre Meloidogyne sp. Asociado al tomate en Nicaragua. Agronomía Mesoamericana, 25(1).

Silva, J. C., Lima-Saraiva, S. R. G., Oliveira-Júnior, R. G., \& Almeida, J. R. G. S. (2013). Modelos experimentais para avaliação da atividade antinociceptiva de produtos naturais: uma revisão. Brazilian Journal of Pharmacy, 94, 18-23.

Soulaidopoulos, S., Sinakos, E., Dimopoulou, D., Vettas, C., Cholongitas, E., \& Garyfallos, A. (2017). Anticholinergic syndrome induced by toxic plants. World journal of emergency medicine, 8(4), 297-301. https://doi.org/10.5847/wjem.j.1920-8642.2017.04.009.

Stillwell, S. B., Fineout-Overholt, E., Melnyk, B. M., \& Williamson, K. M. (2010). Evidence-based practice, step by step: asking the clinical question: a key step in evidence-based practice. The American journal of nursing, 110(3), 58-61. https://doi.org/10.1097/01.NAJ.0000368959.11129.79.

The Royal Children's Hospital Melbourne. Poisoning: Acute Guidelines For Initial Management. (2017). Melbourne: The Royal Children's Hospital. <https://www.rch.org.au/clinicalguide/guideline_index/Poisoning_-_Acute_Guidelines_For_Initial_Management/>

World Health Organization. (2010). Acute intoxication. Geneva: Department of Mental Health and Substance Abuse. <https://www.who.int/substance_abuse/terminology/acute_intox/en/>. 